\title{
Sorbitan tristearate layers at the air/water interface studied by shear and dilatational interfacial rheology
}

\author{
Supporting Information - Interfacial Transport Analysis for the Biconical \\ Disk Rheometer \\ Philipp Erni, Peter Fischer, and Erich J. Windhab \\ Laboratory of Food Process Engineering, \\ ETH Zürich - Swiss Federal Institute of Technology, \\ Schmelzbergstr. 9, 8092 Zürich, Switzerland
}

Often the interfacial flow is assumed to be completely decoupled from the bulk phase flow, that is, dissipation of interfacial stresses into the bulk phases is considered negligible. This approach is suitable if the Boussinesq number

$$
B o=\frac{\eta}{\left(\eta^{(1)}+\eta^{(2)}\right) R_{1}}
$$

is large ( $\eta$ is the interfacial shear viscosity, $\eta^{(i)}$ are the dynamic viscosities of the bulk liquids, and $R_{1}$ is the cup diameter; see Figure 1). In that case, a disk rheometer may be treated as a two-dimensional Couette device, and the interfacial shear viscosity $\eta$ is easily calculated from the torque with the following proportionality:

$$
\eta=\frac{M-\frac{8}{3} R_{2}^{3}\left(\eta^{(1)}+\eta^{(2)}\right) \Omega}{4 \pi R_{2}^{2} \Omega}
$$

where $\Omega$ is the angular velocity of the disk and $R_{2}$ is its radius, with $R_{2} / H_{1} \rightarrow 0$ and $R_{2} / R_{1} \rightarrow 0$. In general, however, a more comprehensive analysis of the flow in the rheometer is desirable, yielding an interfacial velocity distribution that accounts for bulk viscous contributions to the interfacial shear stress.

We consider a three-phase system consisting of two immiscible liquids 1 and 2 with densities $\rho^{(1)}>\rho^{(2)}$ and the interface. The goal is to obtain the velocity distribution $v_{\theta}=f(r, z)$ with $v_{z}=0$ and $v_{r}=0$ by solving the Stokes equations. A cylinder coordinate system $(r, \theta, z)$ with the origin 


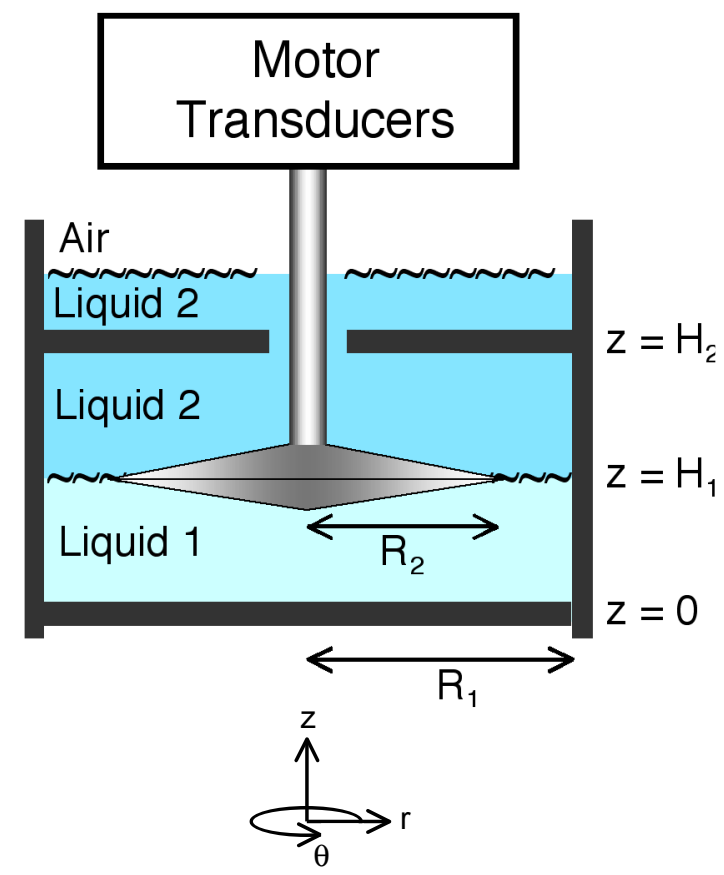

Figure 1: Biconical disk interfacial rheometer. Transducers include torque and normal force transducers and the angular displacement sensor. $R_{1}$ is the inner radius of the cup, $R_{2}$ is the bob radius, $H_{1}$ is the level of the interface. A cylinder coordinate system $(r, \theta, z)$ with the origin in the center of the base plate is used. 
in the center of the base plate of the cup is used. For the analysis, an infinitely flat rotating disk is assumed, and the results are then adapted to a biconical geometry with a few minor corrections. For both bulk phases, conservation of mass and momentum is assumed. In addition, the following balance equations ${ }^{1,2}$ are specified for the interface:

a jump mass balance at the interface

$$
d i v_{\sigma} \mathbf{v}^{(\sigma)}=0
$$

a jump momentum balance at the interface

$$
\mathbf{T}^{(1)} \cdot \mathbf{n}^{(1)}+\mathbf{T}^{(2)} \cdot \mathbf{n}^{(2)}+\operatorname{div}_{\sigma} \mathbf{T}^{(\sigma)}+\rho^{(\sigma)} \mathbf{g}=0
$$

where $\mathbf{v}^{(\sigma)}$ is the interfacial velocity vector, $\mathbf{T}^{(\sigma)}$ is the interfacial stress tensor, $\mathbf{n}^{(1)}$ and $\mathbf{n}^{(2)}$ are the normal vectors perpendicular to the interface pointing into phases 1 and $2, \rho^{(\sigma)}$ is the mass density of the interface and $\mathbf{g}$ is gravity per unit mass. The boundary conditions are: Both bulk liquids and the interfacial layer are incompressible and Newtonian. The velocity distribution and the shape of the interface are independent of time (steady flow), and the interface is assumed to be a plane. Interfacial curvature is neglected, so the normal stress jump does not contain the Laplace pressure. The analysis assumes vanishing Reynolds numbers (Stokes flow). Only insoluble surfactant systems with uniform surface coverages are considered. As such, the interfacial mass balance simplifies to a zero surface divergence term and the tangential stress balance does not include any Marangoni effects. The fluid velocity is zero at the top, bottom and lateral walls and equal to the disk velocity at the edge of the rotating bicone.

Oh and Slattery ${ }^{3}$ have provided an exact solution for the velocity distribution in both liquids and in the plane of the interface. Once this velocity distribution is known, the torque exerted on the disk by both liquids and the interface is calculated according to

$$
\bar{M}=\frac{M}{2 \pi \Omega R_{1}^{3}\left(\eta^{(1)}+\eta^{(2)}\right)}
$$

and

$$
\begin{array}{r}
\bar{M}=\left.\bar{R}_{2}^{3} B o \frac{\partial}{\partial \bar{r}}\left(\frac{\bar{v}_{\theta}^{(\sigma)}}{\bar{r}}\right)\right|_{\bar{r}=\bar{R}_{2}}-\left.\int_{0}^{\bar{R}_{2}} \frac{\partial \bar{v}_{\theta}^{(1)}}{\partial \bar{z}}\right|_{\bar{z}=\bar{H}_{1}} \bar{r}^{2} \bar{r} d r \\
+\left.\frac{1}{Y} \int_{0}^{\bar{R}_{2}} \frac{\partial \bar{v}_{\theta}^{(2)}}{\partial \bar{z}}\right|_{\bar{z}=\bar{H}_{1}} \bar{r}^{2} \bar{r}
\end{array}
$$


where we have used overbars to identify dimensionless quantities. $M$ is the torque exerted on the $\operatorname{disk}^{4}$ and $\bar{M}$ is the reduced torque. $Y=\eta^{(1)} / \eta^{(2)}$ is the bulk viscosity ratio, $\bar{r}=r / R_{1}, \bar{z}=z / R_{1}, \bar{H}_{j}=H_{j} / R_{1}$ and $\bar{v}_{\theta}^{(j)}=$ $v_{\theta}^{(j)} / R_{1} \Omega-\bar{r}$, where $v_{\theta}^{(j)}$ are the $\theta$-components of the velocity distribution in phase $j$. Equation 6 relates $\bar{M}$ to the Boussinesq number, Bo, from which the interfacial viscosity may be obtained. The results for the dimensionless velocity distributions in both liquids and in the plane of the interface are given by

$$
\begin{array}{r}
\bar{v}_{\theta}^{(\sigma)}=-\sum_{i=1}^{\infty}\left[\frac{1}{\xi_{i}^{2}}\left(\frac{A_{i}}{B o}+\frac{1}{Y} \frac{B_{i}}{B o}\right)\right] \\
\times\left[J_{1}\left(\xi_{i} \bar{r}\right)-\frac{\overline{R_{2}}}{\bar{r}}\left(\frac{1-\bar{r}^{2}}{1-\bar{R}_{2}^{2}}\right) J_{1}\left(\xi_{i} \bar{R}_{2}\right)\right] \\
-\frac{\bar{R}_{2}^{2}}{\bar{r}}\left(\xi_{i} \bar{r}\right)-\frac{\overline{R_{2}}}{\bar{r}}\left(\frac{1-\bar{r}^{2}}{1-\bar{R}_{2}^{2}}\right) \\
\bar{v}_{\theta}^{(1)}=\sum_{i=1}^{\infty} A_{i} \frac{\sinh \left(\xi_{i} \bar{r}\right)}{\xi_{i} \cosh \left(\xi_{i} \bar{H}_{i}\right)} J_{1}\left(\xi_{i} \bar{r}\right) \\
\bar{v}_{\theta}^{(2)}=\sum_{i=1}^{\infty} B_{i} \frac{\sinh \left[\xi_{i}\left(\bar{H}_{2}-\bar{z}\right)\right]}{\xi_{i} \cosh \left[\xi_{i}\left(\bar{H}_{2}-\bar{H}_{1}\right)\right]} J_{1}\left(\xi_{i} \bar{r}\right)
\end{array}
$$

where $\bar{r}=r / R_{1}, \bar{z}=z / R_{1}, \bar{H}_{j}=H_{j} / R_{1}$ and $\bar{v}_{\theta}^{(j)}=v_{\theta}^{(j)} / R_{1} \Omega-\bar{r}$ are dimensionless quantities. $J_{1}$ is the Bessel function of the first kind of the first order, $\xi_{j}$ are its zeros and $A_{i}$ and $B_{i}$ are coefficients defined as

$$
\begin{gathered}
A_{i}=\frac{2 \xi_{i}}{\tanh \left(\xi_{i} \overline{H_{1}}\right) J_{0}^{2}\left(\xi_{i}\right)} \cdot\left[\int_{\bar{R}_{2}}^{1} \bar{r} J_{1}\left(\xi_{i} \bar{r}\right) \bar{v}_{\theta}^{(\sigma)}(\bar{r}) d \bar{r}-\frac{1}{\xi_{i}}{\overline{R_{2}}}^{2} J_{2}\left(\xi_{i} \overline{R_{2}}\right)\right] \\
B_{i}=\frac{2 \xi_{i}}{\tanh \left(\xi_{i}\left\{\overline{H_{1}}-\overline{H_{2}}\right\}\right) J_{0}^{2}\left(\xi_{i}\right)} \cdot\left[\int_{\bar{R}_{2}}^{1} \bar{r} J_{1}\left(\xi_{i} \bar{r}\right) \bar{v}_{\theta}^{(\sigma)}(\bar{r}) d \bar{r}-\frac{1}{\xi_{i}}{\overline{R_{2}}}^{2} J_{2}\left(\xi_{i} \overline{R_{2}}\right)\right]
\end{gathered}
$$

where $J_{0}$ and $J_{2}$ are Bessel funtions of the first kind of order zero and two, respectively. Since $A_{i}$ and $B_{i}$ are functions of $\bar{v}_{\theta}^{(\sigma)}$, eq. 7 is an implicit expression for the dimensionless interfacial velocity.

Upon evaluation of the reduced torque $\bar{M}$ from Eq. 6 we need prior knowledge of the dimensionless interfacial velocity distribution, $\bar{v}_{\theta}^{(\sigma)}$. Therefore, 
numerical evaluation of the measured raw data (torque $M$ and angular velocity $\Omega$ ) is necessary, and data acquisition and numerical evaluation of the rheological parameters are carried out separately.

It is more convenient for numerical computation of the interfacial velocity to represent the expressions 7, 10 and 11 derived by Oh and Slattery as the following integral equation of the first kind for $\bar{v}_{\theta}^{(\sigma)}$ :

$$
\overline{r v}_{\theta}^{(\sigma)}(\bar{r})=\bar{R}_{2}^{2} \frac{1-\bar{r}^{2}}{1-\bar{R}_{2}^{2}}+\frac{1}{B o} \int_{0}^{1} K(x, \bar{r}) \bar{v}_{\theta}^{(\sigma)}(x) d x
$$

with the kernel function

$$
K(x, \bar{r})=\frac{1-\bar{r}^{2}}{1-\bar{R}_{2}^{2}} G\left(x, \bar{R}_{2}\right)-G(x, \bar{r})
$$

where

$$
G(x, y)=2 x y \sum_{i=1}^{\infty} \frac{C_{i} J_{1}\left(\xi_{i} x\right) J_{1}\left(\xi_{i} y\right)}{\xi_{i} J_{0}^{2}\left(\xi_{i}\right)}
$$

with $J_{0}$ and $J_{1}$ being Bessel functions of the first kind of orders zero and one, and

$$
C_{i}=\frac{Y}{1+Y} \operatorname{coth}\left(\xi_{i} \bar{H}_{1}\right)+\frac{1}{1+Y} \operatorname{coth}\left(\xi_{i}\left[\bar{H}_{2}-\bar{H}_{1}\right]\right)
$$

This integral equation can be solved with the help of standard numerical methods ${ }^{5}$ : the continuous velocity is replaced by its discrete analog on a $\mathrm{r}$-net, the integral is replaced by a quadrature formula, and the integral equation is then substituted accordingly by a system of linear algebraic equations. The dimensionless total torque in Eq. 7 can be calculated after computation of the interfacial velocity as follows:

$$
\bar{M}=\frac{2 B o}{1-\bar{R}_{2}^{2}}\left[\bar{R}_{2}^{2}+\frac{1}{B o} \int_{0}^{1} G\left(x, \bar{R}_{2}\right) \bar{v}_{\theta}^{(\sigma)}(x) d x\right]
$$

A correction for a small bob angle can be realized easily ${ }^{3}$.

For measurements in oscillation mode, the linear constitutive equation for the interfacial stress tensor is replaced by a complex interfacial fluid model, and the tensor is redefined as:

$$
\mathbf{T}^{(\sigma) *}=\left(\kappa^{*}-\eta^{*}\right) \operatorname{tr}\left(\mathbf{D}^{(\sigma) *}\right) \mathbf{P}+2 \eta^{*} \mathbf{D}^{(\sigma) *}
$$

with $\kappa^{*}=\kappa^{\prime}-i \kappa^{\prime \prime}$ and $\eta^{*}=\eta^{\prime}-i \eta^{\prime \prime}$ where $\kappa^{*}$ is the complex interfacial dilatational viscosity and $\eta^{*}$ is the complex interfacial shear viscosity. $\kappa^{\prime}$ 
and $\eta^{\prime}$ are the viscous and $\kappa^{\prime \prime}$ and $\eta^{\prime \prime}$ the elastic portions, respectively, and $i$ is the imaginary unit. $\mathbf{D}^{(\sigma) *}$ is the interfacial rate of deformation tensor, and $\mathbf{P}$ is a projection tensor that transforms vectors at the interface into their tangential components ${ }^{2}$. After replacing the interfacial viscosities by their complex counterparts defined in Eq. 17, the rest of the analysis is analogous to what we have outlined for measurements in rotational mode. The latter case can then be considered as an oscillatory experiment at a frequency $\omega \rightarrow 0$ and strain amplitude $\gamma_{0} \rightarrow \infty$. In rotation mode the disk rotates with a constant angular velocity $\Omega$, whereas in oscillation mode it oscillates with a constant angular frequency $\omega$ and strain amplitude $\gamma_{0}$. Thus angular velocity is recast as $\Omega(t)=\phi_{0} e^{i \omega t}$ where $\phi_{0}$ is the angular amplitude and $t$ is time. The oscillatory problem is tackled in a slightly different manner in Refs. ${ }^{6-8}$. The method described therein refers to an oscillating cup and a biconical bob suspended by a torsion wire. In that case, the analysis consists of two parts: (i) Calculation of the torque exerted on the sensor and (ii) Calculation of the shape of the oscillation where the torsion constant of the wire must be taken into account. For details we refer to the full analyses published elsewhere ${ }^{6-8}$. In our method ${ }^{9}$ only the torque is calculated, since this is directly the measured property. Both methods lead to identical results. However, the method used in our study leads to integral equations like in the case of rotation, which makes the calculations more straightforward. Another advantage of the method proposed here is that the viscosities of the bulk phase are complex viscosities, thus allowing in principle calculations even for non-Newtonian bulk phases. The method described in the references is based on Newtonian bulk fluids with constant viscosities. Results from dynamic shear experiments will be presented in the complex modulus notation with $G^{*}(\omega)=G^{\prime}(\omega)+i G^{\prime \prime}(\omega)$ where $G^{\prime}$ is the interfacial storage modulus and $G^{\prime \prime}$ is the interfacial loss modulus. They are related to the real and imaginary parts of the complex interfacial viscosity by $G^{\prime \prime}(\omega)=\omega \eta^{\prime}(\omega)$ and $G^{\prime}(\omega)=-\omega \eta^{\prime \prime}(\omega)$.

\section{References}

[1] Edwards, D. A.; Brenner, H.; Wasan, D. T. Interfacial Transport Processes and Rheology; Butterworth-Heinemann: Stonheam, MA, 1991.

[2] Slattery, J. C. Interfacial Transport Phenomena; Springer-Verlag: New York, 1990.

[3] Oh, S.-G.; Slattery, J. C. J. Colloid Interf. Sci. 1978, 67, 516.

[4] Goodrich, F. C.; Chatterchee, A. K. J. Colloid Interf. Sci. 1970, 34, 36.

[5] Press, W. H.; Teukolsky, S. A.; Vetterling, W. T.; Flannery, B. P. Numerical Recipes in C, 2nd ed.; Cambridge University Press: Cambridge, UK, 1992. 
[6] Lee, H. O.; Jiang, T.-S.; Avramidis, K. S. J. Colloid Interf. Sci. 1991, 146, 90.

[7] Nagarajan, R.; Chung, S. I.; Wasan, D. T. J. Colloid Interf. Sci. 1998, 204, 53.

[8] Ray, Y.-C.; Lee, H. O.; Jiang, T. L.; Jiang, T.-S. J. Colloid Interf. Sci. 1987, $119,81$.

[9] Erni, P.; Fischer, P.; Windhab, E. J.; Kusnezov, V.; Stettin, H.; Läuger, J. Rev. Sci. Instrum. 2003, 74, 4916. 